\title{
Deadlock Avoidance in the PNNI Routing Protocol
} A Deadlock Prevention Algorithm

\author{
J Adeane and V W Wittorff \\ School of Electrical \& Computer Engineering, Curtin University of Technology, GPO Box \\ U1987, Perth, WA 6845, Australia
}

\begin{abstract}
The Private Network Node Interface (PNNI), as defined by the ATM Forum, provides a flexible and scalable routing protocol for ATM networks. PNNI routing provides mechanisms for the automatic distribution of aggregated topology information, making use of an artificial hierarchical structure. However, for traffic control schemes that involve the sharing of resources between connections, the PNNI network structure is subject to the problem of deadlock. This paper discusses the deadlock problem in a PNNI hierarchical network and outlines algorithms that serve to prevent the possibility of deadlock.
\end{abstract}

Key words: ATM, PNNI, routing, control, traffic management, deadlock, hierarchical structure, no-loop network, peer group.

\section{INTRODUCTION}

In a digital communication network, there is a need for the provision of two types of services. One is for what is commonly referred to as real-time traffic, for which occasional packet loss is tolerable, but for which both endto-end delay and delay variation must be small. Such a traffic class requires resourcing of bandwidth, and therefore cannot be provided in a connectionless network. We will therefore restrict ourselves to connectionoriented networks.

The second type of service is for non-real-time, or data traffic. Generally network-layer packets comprise a part of the larger packets of higher-layer protocols, and the loss of a single network layer packet causes all of such packets in that higher-layer packet to be discarded and require re- 
transmission. This is highly undesirable, especially since it is often most likely to occur during periods of congestion, when re-transmission will only exacerbate the problem. Packet loss can therefore not be tolerated. However, there are no requirements related to delay and delay variation. Such connections do not need to be resourced, but control is required to ensure that finite buffers cannot overflow. In ATM networks, such control would be provided by the Controlled Cell Transfer Protocol $[1,8]$, in which absolute priority is given to resourced and uncontrolled traffic. Control is exercised on a class or classes of unresourced connections, with no distinction made between connections within the class. In any control scheme where resources (in this case buffer space and associated link credits) are shared between several connections, there is the possibility of deadlock whenever the routing of connections is arbitrary.

Deadlocks may occur when there is a cyclic wait of resources to become available. Of the various types of deadlocks, this report will discuss storeand-forward deadlock. In this case, more than two nodes are involved in the deadlock. For example in a ring topology where the buffer in each node is full of packets awaiting onward transmission via the adjacent node in the ring, but no packets can be sent because all receiving buffers are full.

\section{DEADLOCK PREVENTION}

There are two strategies that can be adopted to prevent deadlocks. The first method is by reserving for each virtual connection (VC) some amount of buffer capacity for the lifetime of the VC. It was found [8] that in this case each VC needs a minimum reservation of a round-trip-delay worth of credits to prevent credit starvation. This strategy has the fatal drawbacks that (i) it requires buffer management and traffic control on a per-VC basis, and (ii) during congestion it leads to intolerably large latencies for all VCs.

The other method to avoid deadlock is by imposing routing constraints. Deadlock configurations can be avoided by placing cuts in the network to form a no-loop network (nolon). A cut is a restriction imposed at a particular switch between two links, which prevents controlled cells that enter the switch on one link from being switched to the other. A no-loop network is a switching network with cuts, such that no circular flows can be switched but all nodes can still be reached by flows.

Wimmer et. al. at the Deutsches Elektronen-Synchrotron (DESY) [7] developed a graph theory formalism in 1978 and proved the following theorems:

Theorem 1: Store-and-forward deadlocks cannot occur in a nolon. 
Theorem 2: $\quad$ It is always possible to find cuts in a switching network, so that the switching network becomes a nolon.

Theorem 3: $\quad$ For each switching network there exists at least one nolon with a minimum number of cuts. This minimum $m$ is

$$
m \geq e-n+1 \text {, }
$$

where $e$ is the number of arcs (links) in the switching network and $n$ is the number of nodes (switches).

Theorem 4: The problem of finding a minimally constrained nolon (with $m$ cuts) is equivalent to correctly numbering the nodes of a switching network.

The equivalence described in Theorem 4 refers to a procedure by which, once the nodes are numbered, arcs are directed (indicated with an arrowhead) in the direction from the node of lower number towards the node of higher number. Cuts are then imposed between any pairs of links at a switch if they are both directed towards that switch, creating a nolon.

This concept of directed arcs does not mean that the links represented by the arcs cannot transmit in both directions, rather the directed arcs effectively impose a hierarchical switching structure on the network. In this hierarchy, flows can only go up-up (against the arrows on successive hops), down-down (with the arrows on successive hopes), or up-down (against an arrow on one hop and then with an arrow on the next). This ensures circular flows are impossible.

Figure 1 shows an example of a switching network that contains loops and so in which cuts are required for it to become a nolon. The numbering of the nodes that is shown gives arcs that are directed so as to require only four cuts, yielding a minimally constrained nolon according to Theorem 3 above. The cuts are indicated by a line segment cutting a node boundary in-between the two links involved.

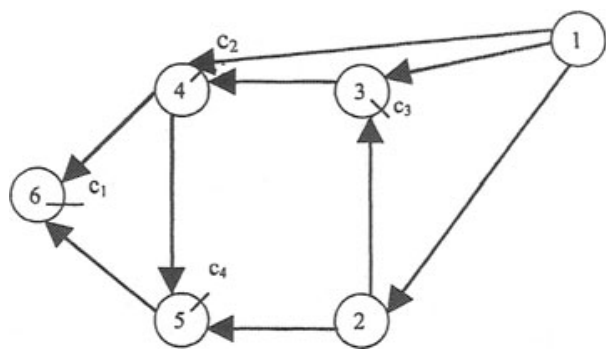

Figure 1. A minimally constrained no-loop network configuration. 
In Figure 1, the cut $c_{1}$ at node 6 prevents traffic from traversing the path 4-6-5 or 5-6-4. The cut $c_{2}$ prevents routing between nodes 1 and 3 via node 4 , and so on.

So to avoid deadlocks in a network, cuts can be placed to eliminate possible routes for controlled connections that may lead to a deadlock configuration. There are costs involved, however, for having reduced connectivity. The packets on a connection might have to travel a longer distance, using more network resources, or they might have to use a lower capacity link. A minimum number of cuts will tend to minimise the cost involved, and therefore the task is to develop an efficient algorithm that numbers the nodes to produce a minimally constrained nolon, or close to it. We have developed an algorithm for this purpose, which will not be discussed in this paper.

This paper will be devoted to the PNNI hierarchical framework, and how to implement an algorithm for the determination of cuts within such a hierarchical framework.

\section{PNNI}

\subsection{The PNNI Hierarchical Framework}

In a PNNI topological structure, a system may consist of physical switches or an interconnected group of switching systems that form a virtual switching structure. A physical switch at the lowest hierarchical level is called a lowest level node. The nodes are organized into peer groups. A peer group (PG) is a group of interconnected nodes with an arbitrary number of common prefix addresses. The peer group identifier (ID) is based on the switch's ATM address and thus is automatically generated.

A peer group leader (PGL) is elected for each peer group. It can be any node in the network, depending on its leadership value. The system administrator can configure priorities for the PGL election, for example a node with more processing power can be given higher leadership value. The role of a PGL is to summarize topology and state information about the peer group it represents and passes the information up the hierarchy. When it receives summarized information about other peer groups, it passes the information down the hierarchy to its child nodes. Thus the PGL participates in topology exchange protocols at higher level of the hierarchy. It can be seen as a single logical group node (LGN) (a virtual switch) in the next level of the hierarchy. At the lowest level, a peer group is a number of physically interconnected switches with some common leftmost address bits. At higher 
levels, LGNs are interconnected by logical links, which represent one or more links between nodes at the lower level. Addressing of LGNs reflects the hierarchical structure, e.g. node X.Y.2 is a member of peer group X.Y.

All nodes in a peer group have the same topology information, that is each node, link, and advertised reachable address prefix. Nodes do not see a complete topology of other peer groups. In Figure 2, node X.Y.1 sees nodes X.Y.2, X.Y.3, X.Y.4, and all links between them, but it does not see any of the nodes or links in the adjacent peer group, e.g. X.Z.1.

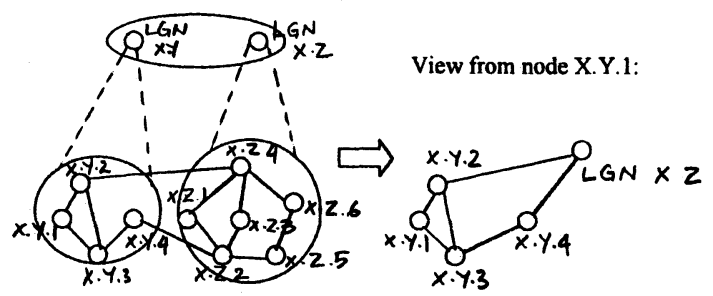

Figure 2. Concept of hierarchy in PNNI.

\subsection{The PNNI Protocol Mechanisms}

\subsubsection{The Hello protocol}

Nodes periodically exchange Hello packets that allow them to determine the up-to-date operational status and hierarchical structure of their neighbours. Hellos received from the neighbour indicate that the neighbouring switch is in active state. The Hello packets contain the node's ATM end system address, node ID, peer group ID, and link status information. When a node receives a Hello packet from its neighbour, it compares the peer group ID received with its own peer group ID to determine whether they are in the same peer group.

\subsubsection{Database synchronization}

When a node is first activated, it does not have the topology information of the network. It only has information about its own state and therefore it cannot compute routes or originate calls. As soon as a link between two nodes is activated (according to the Hello protocol), the two nodes execute a protocol to synchronise their topology databases. Each node sends the other a summary of its topology information. 


\subsubsection{Flooding of PNNI Topology State Elements (PTSEs)}

Topology information changes due to establishment of new links, link failure, changes in reachability information, and changes in QoS parameters. The flooding mechanism updates each member's view of the network topology periodically. While database synchronization is limited to the moment where two neighbours learn about their existence, flooding lasts as long as the network is up and running.

\subsection{Prevention of Deadlock in the PNNI Protocol}

Deadlocks can possibly occur in PNNI networks, within a peer group and also between peer groups. Since PNNI topology is based on an artificial hierarchical structure, whereby the hierarchy is an abstraction and only for the sake of scalable routing, in one view the topology can be seen as a flat network for the purpose of creating a single nolon. Thus deadlock avoidance can be achieved by placing cuts in a single physical network (the aggregated lowest level in the PNNI hierarchy).

In this view, to decide on the cuts to be placed in the network, the entire network must be considered, and all nodes numbered 1 to $n$ to give a minimally constrained (or near-minimally constrained) nolon. This is simply not scalable across an entire network, and furthermore the cuts must frequently be reconfigured network-wide when any single node or link fails, or any other topological changes are made. An alternative implementation is considered in the next section.

Whatever the implementation, whether global or local, for efficiency and network stability the cuts should be re-determined only as often as necessary. This would be after database synchronization (initialization) and every time there is a change in the physical topology. However the cuts need not be re-determined in response to a change in QoS parameters, e.g. available bandwidth.

\subsection{Use of the PNNI Hierarchy in Determination of Cuts}

The approach that will be outlined in this paper makes use of the abstract hierarchical structure of PNNI network to determine the routing constraints. There are two types of cuts to be implemented, within a peer group and between peer groups. In the PNNI framework, the network at all but the lowest hierarchical level is considered as a group of logical group nodes. The cuts placed between logical group nodes are not actually physical cuts, since the links between logical group nodes are artificial links that represent physical connections at the lowest level. The cuts thus determined have to be 
implemented in the physical level. Thus to determine all of the cuts required in the physical level, one has separately to analyse each peer group (PG) at every layer of the PNNI hierarchy. High-level cuts, which are not real, must be cascaded down the hierarchy until they are implemented in the physical level.

For our purposes, it is necessary to retain in higher-layer PGs, multiple representative links between LGNs, one for each link between the PGs in the lower-layer represented by the two LGNs. An example is P.G.A. in Figure 3. The two links between LGNs A2 and A4 should not be collapsed to a single link.

Using this distributed implementation, the cuts required within each peer group at every level of the hierarchy can be independently determined, and in no particular order. Changes in the topology solely within one peer group necessitates re-evaluation of the cuts required within that peer group, but does not affect the cuts required in any of the other peer groups in the same layer of the hierarchy. If a link that crosses a peer group boundary is affected, then of course that is reflected in a change in the topology of the parent peer group in the next-higher level of the hierarchy, and so the cuts there need re-evaluation, but not elsewhere.

Whenever cuts are evaluated or re-evaluated in a peer group that is not in the physical level, these must be implemented in the relevant peer groups in the next-lower hierarchical level. However it is important to note that in our implementation these latter cuts will always involve external links (links that cross the peer group boundary), and so will not interfere with the cuts within the lower-level peer group, which will only be between internal links.

This method is highly scalable, since it fully utilizes the abstraction provided by the PNNI hierarchical structure. Also, by implementing the cuts at every level and translating each individual cut to the lower level, the dependency on reliability of topology information from switches belonging to other peer groups has been reduced significantly.

\section{IMPLEMENTATION IN A HIERARCHY}

The implementation is as follows:

For each peer group, whenever the topology of the peer group changes:

1. Number the nodes within the peer group so as to create a minimally constrained nolon.

2. If the peer group under consideration is at the physical level, the cuts are real and the process for this peer group is complete. Otherwise 
the cuts are abstract and must be cascaded down the hierarchy, so for each cut so determined:

3. Consider the peer group in the next-lower level that comprises the LGN in which the cut is to be imposed. If the two external links terminate at the same node in this peer group, place a cut between them in this node. Otherwise choose the node that terminates the smaller number of internal links, and place cuts in this node between the external link and each of the internal links, but not between any two of the internal links.

4. Repeat from step 2.

Let us consider an example network with three levels of hierarchy, numbered from 2 (the highest level) down to 0 (the lowest):

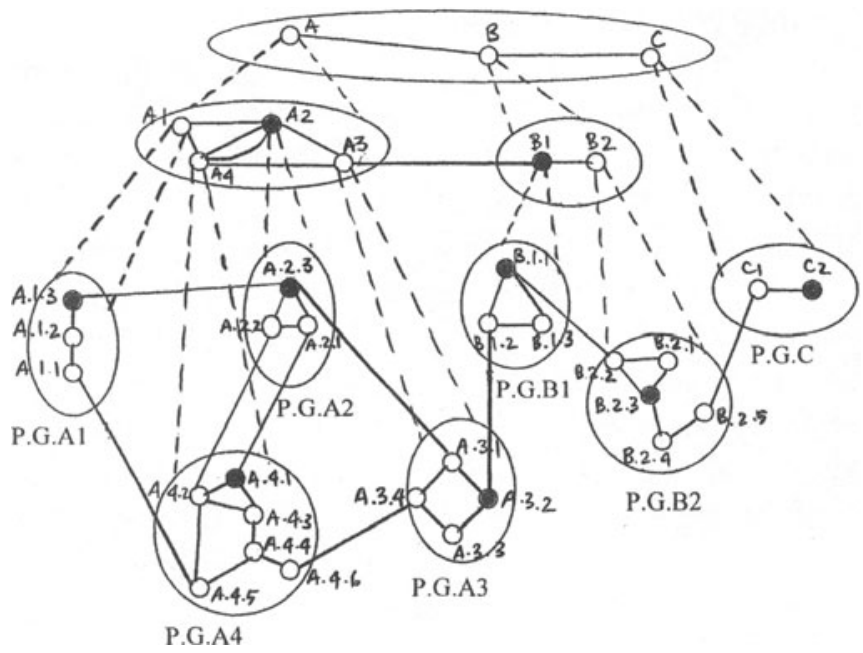

Figure 3. Hierarchical network structure

There is no need to do so, but let us start at level 2:

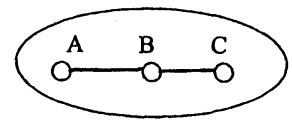

Figure 4. The highest hierarchical level

Since LGN A, B, C altogether constitute a nolon configuration, we can consider the children nodes inside LGN A, followed by LGN B and LGN C. 
Consider next Level 1:

\section{LGN A:}

We have to ensure that P.G.A constitutes a nolon configuration by numbering the nodes and imposing cuts between each pair of directed arcs coming into one node.

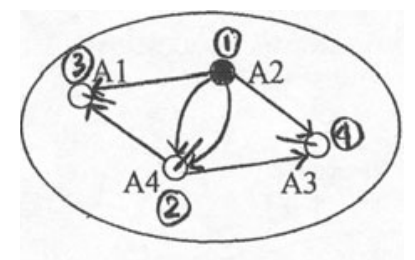

Figure 5. A nolon for P.G.A.

In the above configuration, there are three cuts to be implemented in P.G.A, which consists of six links and four nodes (consistent with the minimum number of cuts achievable). A1, A2, A3, A4 are not in the physical level (level 0). Therefore the cuts among them are abstract and they have to be cascaded to the next-lower level.

\section{Consider now LGN A1.}

LGN A1 has one cut in level 1. Thus we draw peer group A1 in terms of its members and abstract the nodes where the two directed arcs come from as a single node.

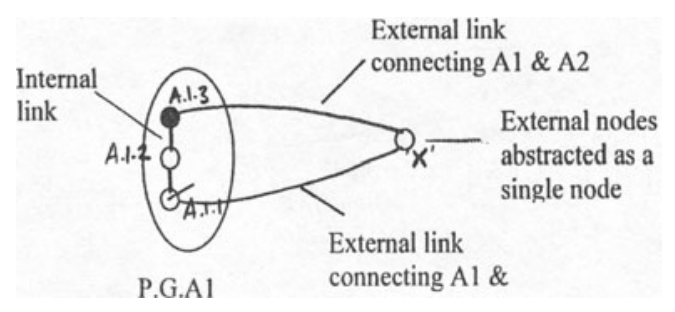

Figure 6. Peer group A1 and its external links

The border nodes are A.1.1 and A.1.3. In our implementation we only consider the border nodes for implementation of these cascaded cuts so that the cut does not disconnect a PG member from the rest of the group, and also so that these cascaded cuts do not interfere with independently-determined internal cuts.

We place a cut to prevent the internal-external loop, arbitrarily in node A.1.1., which puts the cut in the physical level. 
Consider now LGN A3:

There is a cut in LGN A3 between A2-A3-A4 in level 1. Since A3 is not in level 0 , we redraw $A 3$ in terms of its PG members and abstract the external links from A2 and A4 as coming from a single external node.

There are 2 border nodes associated with the cut in LGN A3, which are A.3.1 and A.3.4. Since A.3.1 and A.3.4 each has two internal links, we can place the cut in either A.3.1 or A.3.4. Let us place the cut in A.3.1.

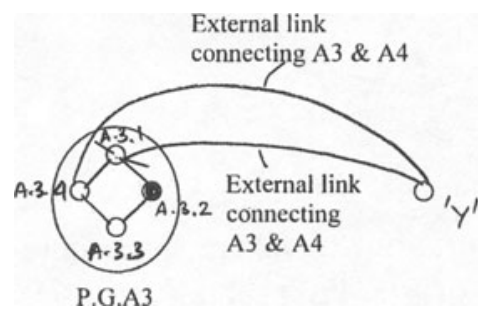

Figure 7. Peer Group A3 made free of internal-external loops

The final Level 1 node in P.G.A. is LGN A4:

There is a cut in LGN A4 at level 1 between A2-A4-A2. There are two border nodes, A.4.1 and A.4.2. A.4.1 has two internal links and A.4.2 has three internal links. Since A.4.1 has the fewer number of internal links, we put two cuts at A.4.1 between the external link and each of the two internal links.

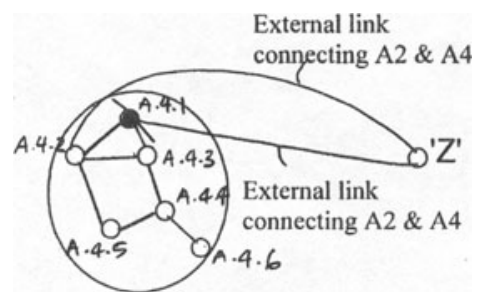

Figure 8. Peer Group A4 is made free of internal-external loops

The other Level 1 peer group, P.G.B. is already a nolon.

Now level 0:

Let us consider for example P.G A.4. When it is correctly numbered, it is found that two cuts are required to make it a nolon. 


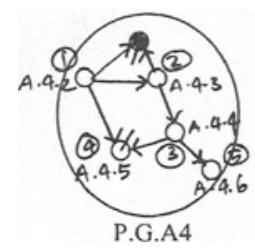

Figure 9. A nolon for P.G.A.4.

The algorithm is repeated for all other peer groups in this layer, producing the final system of cuts shown below:

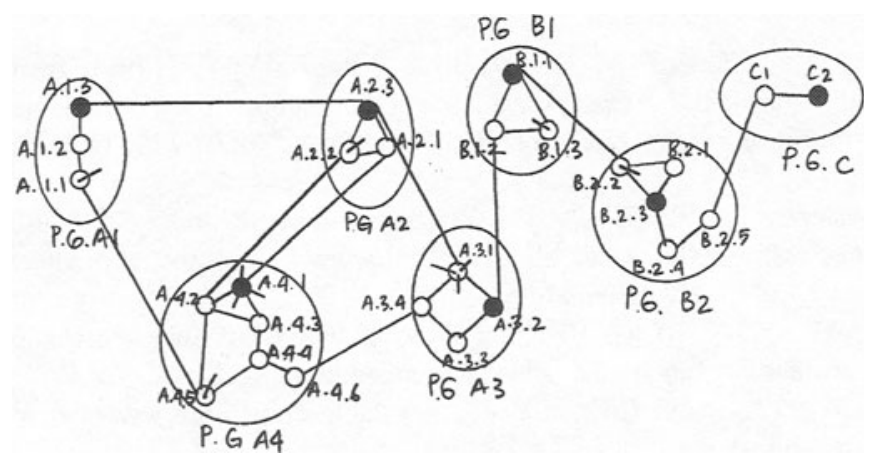

Figure10. The physical layer showing all cuts required

For this example network, which consists of 34 links and 26 nodes, the number of cuts needed at physical level is 11 .

\section{CONCLUSION}

In any control scheme where resources (for example buffer space and associated link credits) are shared between several connections, there is the possibility of deadlock whenever the routing of connections is arbitrary. PNNI networks are subject to deadlock within peer groups and between peer groups. Deadlock avoidance can be achieved by placing a number of cuts to prevent traffic being switched between two links. This paper outlines a method of implementing the routing constraints hierarchically, making use of the structure of PNNI network. The artificial hierarchical structure that provides information abstraction is fully utilized to allow faster processing time and higher reliability. Each group of switches can now construct a no- 
loop network configuration without having to know the entire network topological structure.

\section{ACKNOWLEDGEMENTS}

This work was supported by research funds from the Advanced Materials and Networking Group, within the School of Electrical \& Computer Engineering, Curtin University of Technology.

\section{REFERENCES}

[1] Budrikis, Z.L., Cantoni, A, Hullett, J.L., Wittorff, V.W. (1995). Switching Protocol Providing Controlled Access to Available Asynchronous Network Service, International Patent Publication No. WO96/03824, US Patent App. No. 08/776237, Priority date 25 July 1994.

[2] Cisco Systems, Inc. (2001). "ATM Routing with IISP and PNNI" in Guide to ATM Technology, May 2001. Available at: [http://www.cisco.com/univercd/cc/td/doc/product/ atm/c8540/12_0/13_19/atg/pnni.htm]

[3] CISE (1997). CIS 777 LAB II: PNNI Routing, The Ohio State University. Available at: [http://www.netlab.ohio-state.edu/cise/download.htm]

[4] Gaude, L (1997). PNNI Control Point. Available at: [http://www.networking.ibm.com/ pnni/pnniwp.html]

[5] Gunther, K.D. (1981). "Prevention of Deadlocks in Packet-Switched Data Transport Systems" in IEEE Transactions on Communications, Vol.Com 29, No.4, April 1981.

[6] The ATM Forum Technical Committee Private Network-Network Interface Specification Version 1.0 (PNNI 1.0), af-pnni-0055.000, March 1996.

[7] Wimmer, W. (1978). Ein Verfahren zur Verhinderung von Verklemmungen in Vermittlernetzen, DESY DV-78/05, October 1978 (translated by Budrikis, Z.L.).

[8] Wittorff, V.W. (1995). Controlled Cell Transfer Protocol, ATRI Networking Research Laboratory Seminar, document library code NRL-SL-013, 7 September 1995. Available from: [V.Wittorff@ece.curtin.edu.au]

[9] Wittorff, V.W. (1995). Switch Design Implementing Controlled Cell Transfer, ATRI Networking Research Laboratory Technical Memorandum, document library code NRLTM-079, 4 May 1995. Available from: [V.Wittorff@ece.curtin.edu.au] 